\title{
Some considerations of effects-induced errors in resonant cantilevers with the laser deflection method
}

\author{
Hamed Sadeghian ${ }^{1,2}$, Chung-Kai Yang ${ }^{2}$, Khashayar Babaei Gavan ${ }^{3}$, \\ Johannes F L Goosen ${ }^{2}$, Emile W J M van der Drift ${ }^{3}$, \\ Herre S J van der Zant ${ }^{3}$, Andre Bossche ${ }^{2}$, Paddy $\mathbf{J}$ French $^{2}$ and \\ Fred van Keulen ${ }^{1}$ \\ ${ }^{1}$ Structural Optimization and Computational Mechanics (SOCM) Group, Department of Precision and \\ Microsystems Engineering, Delft University of Technology, The Netherlands \\ ${ }^{2}$ Electronic Instrumentation Laboratory, Microelectronics Department, Delft University of Technology, \\ The Netherlands \\ ${ }^{3}$ Kavli Institute of Nanoscience, Delft University of Technology, Delft, The Netherlands \\ E-mail: H.SadeghianMarnani@tudelft.nl and Hamed.Sadeghian@gmail.com
}

Received 15 July 2010, in final form 20 August 2010

Published 29 September 2010

Online at stacks.iop.org/JMM/20/105027

\begin{abstract}
Micro/nano resonant cantilevers with a laser deflection readout have been very popular in sensing applications over the past years. Despite the popularity, however, most of the research has been devoted to increasing the sensitivity, and very little attention has been focused on effects-induced errors. Among these effects, the surface effects and the so-called readout back-action are the two most influential causes of errors. In this paper, we investigate (1) the influence of the surface effects such as water adsorption, gas adsorption, and generally surface contaminations, and (2) the effect of the laser deflection detection, including power and positions of the laser, on the resonance frequency of silicon cantilevers. Our results show that both the surface contaminations and the laser back-action effects can significantly change the resonant response of the cantilevers. We conclude that the effects have to be taken into account, particularly in the case of ultra high sensitivity cantilevers.
\end{abstract}

(Some figures in this article are in colour only in the electronic version)

\section{Introduction}

Microelectromechanical silicon cantilever resonators have been used in a wide range of sensor applications [1-7]. Because of their ultra-sensitivity to small changes, they have attracted considerable interest in the last few years. The basic principle of sensing the small changes is the measurement of the resonance frequency change due to added mass or stiffness change. Unsurprisingly however, as the sensitivity increases, small mass or stiffness changes due to nonspecific bindings such as surface contaminations (e.g. water adsorption, gas adsorption and oxidation), and also effects inducing heating, viscosity or humidity that were insignificant in previous lowresolution sensors $[8,9]$ can no longer be neglected in today's ultra-sensitive cantilevers.
In typical mass sensing experiments nowadays, the sensing cantilever is exposed to the environment of interest (e.g. molecule solution) and dried with techniques involving different solutions [10]. The drying process and the transportation between solutions may result in increased nonspecific binding. Because the cantilever resonators are extremely sensitive to small changes and can be vulnerable to other external disturbances and effects, the specificity of the cantilever is therefore severely degraded by nonspecific adsorption and by contamination during the dipping and drying steps $[1,11]$. Consequently, a major challenge is to distinguish between frequency variations caused by specific mass change and frequency variations caused by environmental changes.

Besides environmental influences, the back-action effects from the resonance frequency detection can also affect 
cantilever behavior and cause frequency variations. Various readout techniques have been used to measure the cantilever resonance frequency [12-14]. Among them, the laser deflection technique $[15,16]$ has been shown to be sensitive for detection of extremely small motions, achieving measurements of sub-attonewton forces [2] and chemical and biological matter ranging from attogram to zeptogram masses $[11,17]$. It is a robust detection method with high sensitivity and can be applied to different kinds of cantilevers in different environments [1,11,17-23]. Despite its popularity, however, the back-action from the laser on the resonant cantilever remains an issue. It is commonly assumed that the influence of the laser on the resonant sensor device can be neglected, but as we will show in this work, when resonators such as cantilevers become more sensitive, they also become more susceptible to disturbances caused by the laser beam.

Therefore, the goals of the present work are as follows. (1) Studying the effect of the surface contamination on the resonance response of the sub-micron cantilevers. We show that the mass and the stiffening effects of the contaminations can cause significant shifts in the resonance frequency of the ultra-sensitive cantilevers. (2) Investigating the effect of the readout back-action, focusing on the laser deflection detection technique, and its effect on frequency variations of the resonant cantilevers.

\section{Method and approach}

\subsection{Silicon cantilevers}

In order to understand the influences of the environment and laser detection back-action, cantilevers of different thicknesses $(1019,340,93 \mathrm{~nm})$ are used in this work. Measurements on these cantilevers help verify the effects of the contamination and back-action, on different cantilever sensitivities and a surface-to-volume ratio from bulk to ultrathin. Meanwhile, single-crystal silicon is used because it is one of the most common cantilever materials, with well-known crystal properties, characteristics and stable fabrication processes. This helps minimize material and process variations between different cantilever process batches, and helps the comparison of contamination and back-action effects studied in this paper.

All silicon cantilevers measured in this work were fabricated from (1 000$)$ Smartcut $^{\circledR}$ silicon-on-insulator wafers with $1 \mu \mathrm{m}$ buried oxide and a $340 \mathrm{~nm}$ silicon device layer. For the $93 \mathrm{~nm}$ thick cantilevers, the sample wafer was oxidized thermally and etched in a HF solution to reduce the device layer thickness; for the $1019 \mathrm{~nm}$ thick cantilevers, single-crystal silicon was grown on sample wafers by epitaxy, cross-section of the interface between the original surface and the grown layer was checked by SEM and revealed a smooth continuation of the growth. Final thicknesses of all sample wafers were determined by ellipsometry measurements fitted with a SOI wafer multi-layered model. All cantilevers were patterned by standard photolithography followed by $\mathrm{SF}_{6}$ plasma etching on the wafers. The patterned structures were underetched in a HF solution and released using the critical point drying technique. Figure 1 shows the SEM pictures of the fabricated cantilevers. In order to check the smoothness of the cantilevers' surfaces to ensure minimal roughness-induced surface stress, surface loss and sensitivity effects for later experiments [24, 25], the cantilevers' surfaces were measured by AFM. The results confirm that the roughness of all cantilevers is sub-nm; therefore, the surfaces can be considered smooth and the roughness effects can be neglected. Figure 2 shows the AFM measurements of the $1 \mu \mathrm{m}$ thick cantilever.

\subsection{Optical detection method and the measurement setup}

With the introduction of scanning probe techniques like AFM, several amplitude detection methods have been proposed to measure the resonance frequency of microcantilevers with subAngstrom sensitivity, of which the optical lever method is most often used. The optical method involves a focused laser beam at the cantilever surface, and the beam is then reflected back onto a set of photo-diodes, where the cantilever motion is sensed by monitoring the displacement of the laser beam on the diode.

The schematics of the setup used are depicted in figure 3. The deflection of a $658 \mathrm{~nm}$ laser light from the free end of a cantilever generates a voltage difference on a twosegment photo-diode. This time-varying voltage difference is analyzed by a spectrum analyzer to measure the thermal noise spectrum. The diameter of the laser spot is estimated to be $6 \mu \mathrm{m}$ and a power of $1.3 \mathrm{~mW}$ is typically used during measurements in vacuum. The electronic bandwidth of the setup is $5 \mathrm{MHz}$ and its sensitivity is estimated to be $1 \mathrm{pm}$ $\mathrm{Hz}^{-1}$. The resonance frequency of a cantilever is found by fitting a Lorentzian curve to the amplitude-frequency measurements. The curve peak is taken as the resonance frequency.

The optical lever method is a non-contact measurement system that does not need extra layers or electrodes for electric contacts. This greatly simplifies the cantilever structures and works on most materials. Although it cannot be integrated, the method is nevertheless highly robust and works in vacuum, gas or liquid environments. To avoid any unwanted effects caused by cantilever actuation, we have not used external actuations. Further details were reported in a previous paper [26].

\subsection{Cantilever theory}

The cantilever is assumed to deform in the linear elastic regime. As shown in the schematic (figure 4), the $x$ - and $y$-axes are assumed to be oriented parallel to the length and the width of the cantilever, respectively, and the $z$-axis is directed upward, perpendicular to the cantilever surface. The origin being located at the center of the cross-section and coincides with the clamped end.

The resonance frequency depends on mass and stiffness and for a cantilever it follows [26]

$$
f_{0}=\frac{(1.875)^{2}}{2 \pi \sqrt{12}} \frac{t}{L^{2}} \sqrt{\frac{E}{\rho}},
$$

where $E$ is Young's modulus, $L$ is the length, $t$ is the thickness and $\rho$ is the density of the cantilever. Changes in either the 

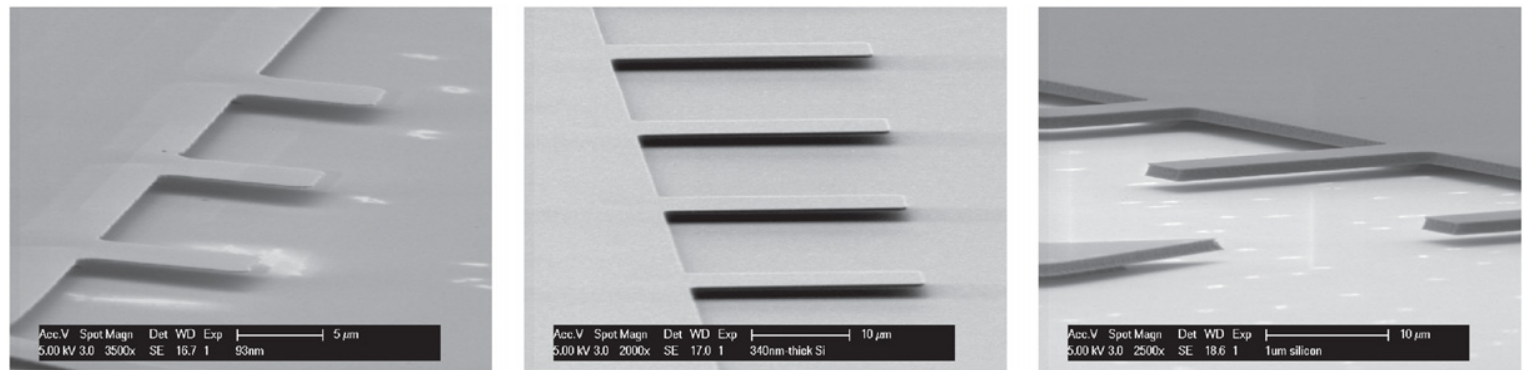

Figure 1. SEM of measured cantilevers of various dimensions. From left to right: $93 \mathrm{~nm}, 340 \mathrm{~nm}$ and $1019 \mathrm{~nm}$ thick cantilevers.
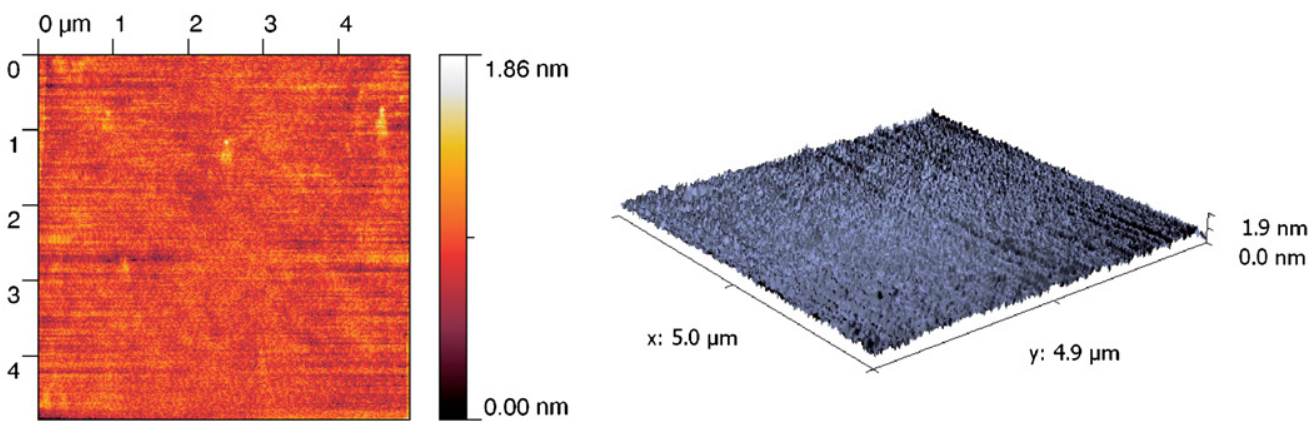

Figure 2. AFM measurement of the epitaxy $1 \mu \mathrm{m}$ silicon surface. The smooth surface indicates continuous single crystal growth from the original surface. The roughness measured has a root mean square of $0.155 \mathrm{~nm}$.

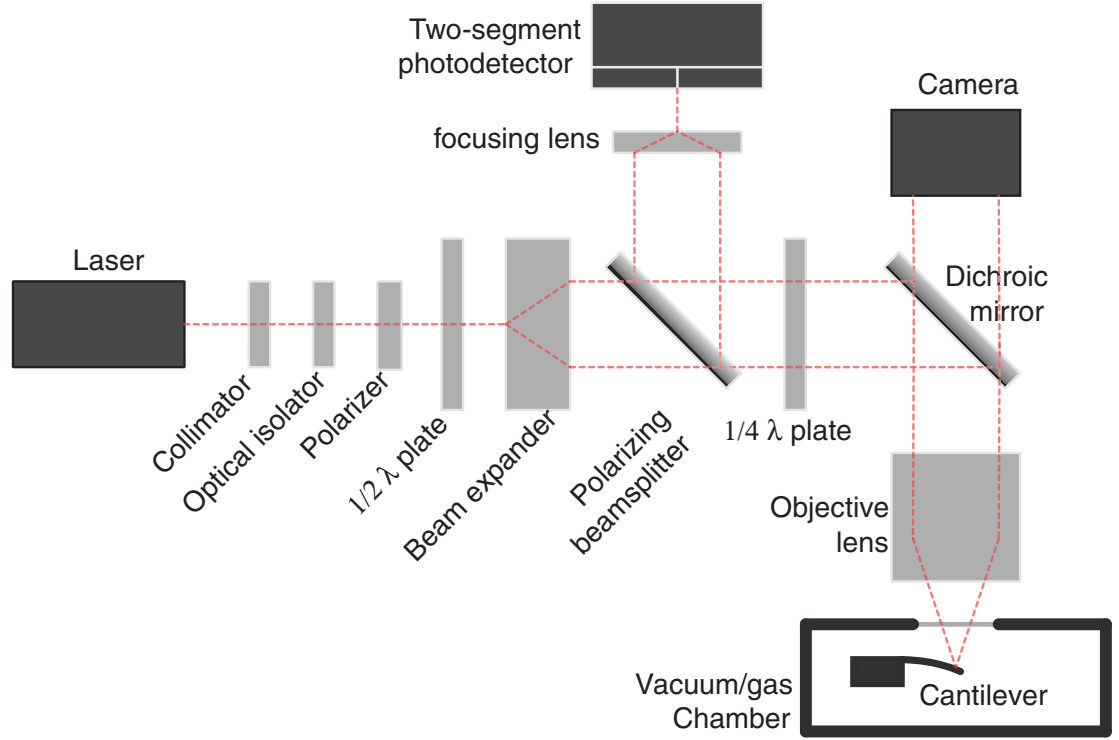

Figure 3. Schematic of the optical deflection setup.

mass or the stiffness of the cantilever would also cause shifts in the resonance frequency.

In order to check the validity of the fabricated cantilevers, resonance frequencies of the cantilevers in various dimensions were measured. Figure 5 shows the resonance frequency data versus length for a set of $340 \mathrm{~nm}$ thick cantilevers, measured in air at 1 bar, and in a vacuum of $10^{-6}$ mbar. The measurements confirmed the relation in equation (1) between the resonance frequency and the length of the cantilevers. A curve is fitted via equation (1) to the experimental data (the dashed line in figure 5).

\section{Experimental measurements and discussions}

In the following sections, the effect of surface contamination and laser back-action on the resonance frequency and its stability in air over time are studied.

\subsection{Surface contamination}

If the surface of the cantilever is clean without any contamination such as adsorbed water and gas molecules, then the adsorbed mass of the targeted molecules can be calculated 


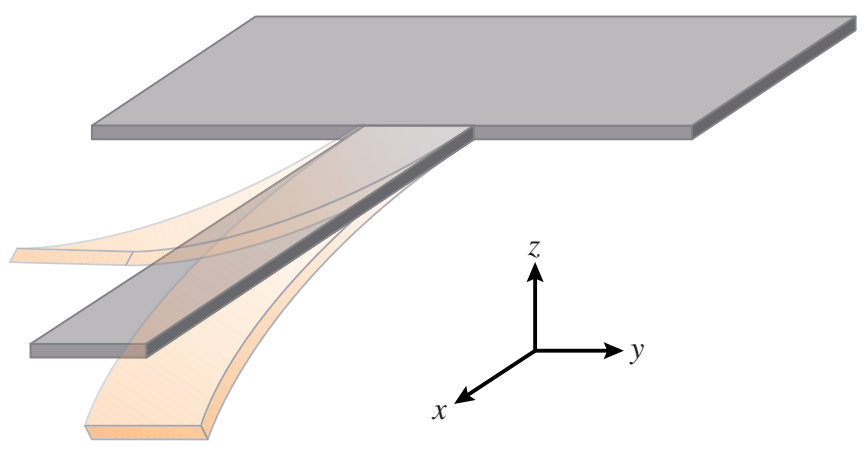

Figure 4. Schematic of the cantilever.

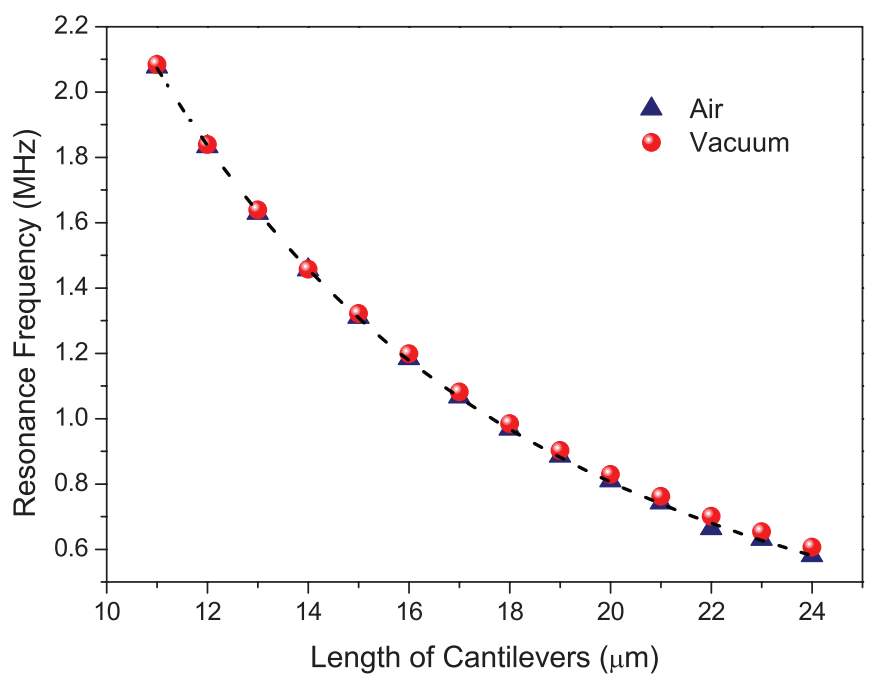

Figure 5. The resonance frequency versus length of the cantilever in air and in vacuum. The difference between the air and the vacuum measurements for the longer cantilevers is larger because of the air damping and mass loading effect. The dashed line shows the fitting curve.

directly from the resonance frequency shift. Assuming that the adsorbed mass is distributed evenly along the cantilever and that the stiffness change is negligible (in the spirit of previous papers $[27,28])$, the mass change can be calculated using

$$
\Delta m=-2 \frac{m}{f_{0}} \Delta f
$$

where $\Delta m$ is the change in the effective mass, $m$ is the effective mass of the cantilever and $\Delta f$ is the resonance frequency shift due to the added mass. However, working in air or other uncontrolled environments can cause unwanted gas and water adsorption, and other surface contaminations, which will result in additional significant frequency shifts [29]. Therefore, the calculated adsorbed mass does not accurately represent the real adsorbed mass of interest. To experimentally show the effect of ambient contamination, we analyzed the resonance frequency shift of cantilevers by the procedure described below.

(i) The resonance frequencies of the cantilevers were measured in ambient air, which we refer to as the frequency before vacuum $\left(f_{\text {bv }}\right)$. The fabricated devices were first purposely left in atmospheric conditions to allow adsorption of different gases and water molecules, as well
Table 1. The resonance frequency of cantilevers of different dimensions in air and vacuum conditions. $f_{\mathrm{bv}}, f_{v}$ and $f_{\mathrm{av}}$ represent the resonance frequencies before vacuum, vacuum and after vacuum, respectively. The resonance data are in $\mathrm{kHz}$.

\begin{tabular}{clllc}
\hline$L \times w \times t\left(\mu \mathrm{m}^{3}\right)$ & $f_{\mathrm{bv}}$ & $f_{v}$ & $f_{\mathrm{av}}$ & $f_{\mathrm{av}}-f_{\mathrm{bv}}$ \\
\hline $96 \times 8 \times 1.019$ & 123.65 & 147.968 & 128.85 & 5.2 \\
$24 \times 8 \times 0.340$ & 583.73 & 607.108 & 585.48 & 1.75 \\
$8 \times 8 \times 0.093$ & 994.53 & 1090 & 973.6 & -20.93 \\
\hline
\end{tabular}

as native oxide growth. The adsorption and oxidation were left to saturate over a period of a few days before the resonance frequencies of the cantilevers were measured in air. At this stage of the experiment, masses and stiffness from surface adsorptions and oxidations are added to the newly fabricated cantilever and are referred to as the added mass-stiffness effects.

(ii) The cantilevers were then loaded into vacuum $\left(10^{-6} \mathrm{mbar}\right)$ for two days to allow degassing and desorption at the surfaces of the cantilever (reduced added mass-stiffness effects). Then the resonance frequencies of the cantilevers were measured in vacuum $\left(f_{v}\right)$. The difference between these resonance frequencies and those measured in step 1 $\left(f_{\text {bv }}\right)$ is dominated by the air mass loading effect.

(iii) The vacuum chamber was vented with ambient air and the resonance frequencies were measured immediately after the pressure reached atmospheric pressure $\left(f_{\text {av }}\right)$. The measurements were done within a few minutes in order to ensure minimal re-adsorption of water and contaminants, while providing the same amount of air mass loading compared to the measurements in step 1.

This procedure was repeated for 1019, 340 and $93 \mathrm{~nm}$ thick cantilevers. With the above procedure, the frequency shift due to additional native oxide growth on silicon and the shift due to air damping were minimized. Figure 6 shows the resonance frequencies of a cantilever $93 \mathrm{~nm}$ thick, $8 \mu \mathrm{m}$ wide and $8 \mu \mathrm{m}$ long. A $20.9 \mathrm{kHz}$ resonance frequency shift was observed between the resonance frequency before and after vacuum. For thicker cantilevers, the shifts are less than that of the $93 \mathrm{~nm}$ thick one. The resonance frequency shifts due to the surface contamination strongly depend on the cantilever thickness because thicker cantilevers are less sensitive to the surface contamination effects, due to their lower surface-tovolume ratio. Table 1 shows the resonance data corresponding to steps 1-3 from cantilevers of different dimensions.

As an example, from equation (2) the equivalent adsorbed mass of the $21 \mathrm{kHz}$ shift in the $93 \mathrm{~nm}$ thick cantilever is estimated to be $128 \mathrm{fg}$, and is in the same order of magnitude as the measured mass (500 fg) in [30]. Moreover, the cantilevers presented in this work have a sensitivity of about $6.5 \mathrm{ag} \mathrm{Hz}^{-1}$. Here it is shown that the ultra-sensitivity of a cantilever can be easily compromised by non-specific surface contaminations. Therefore, high resolution mass sensing can only be achieved if the contaminations are kept low, or compensated.

It is also important to note that the estimated $128 \mathrm{fg}$ is not the real absorbed mass, but rather the effective mass that is derived from the shift in resonance frequency. This shift, however, is caused by the combination of both the additional 


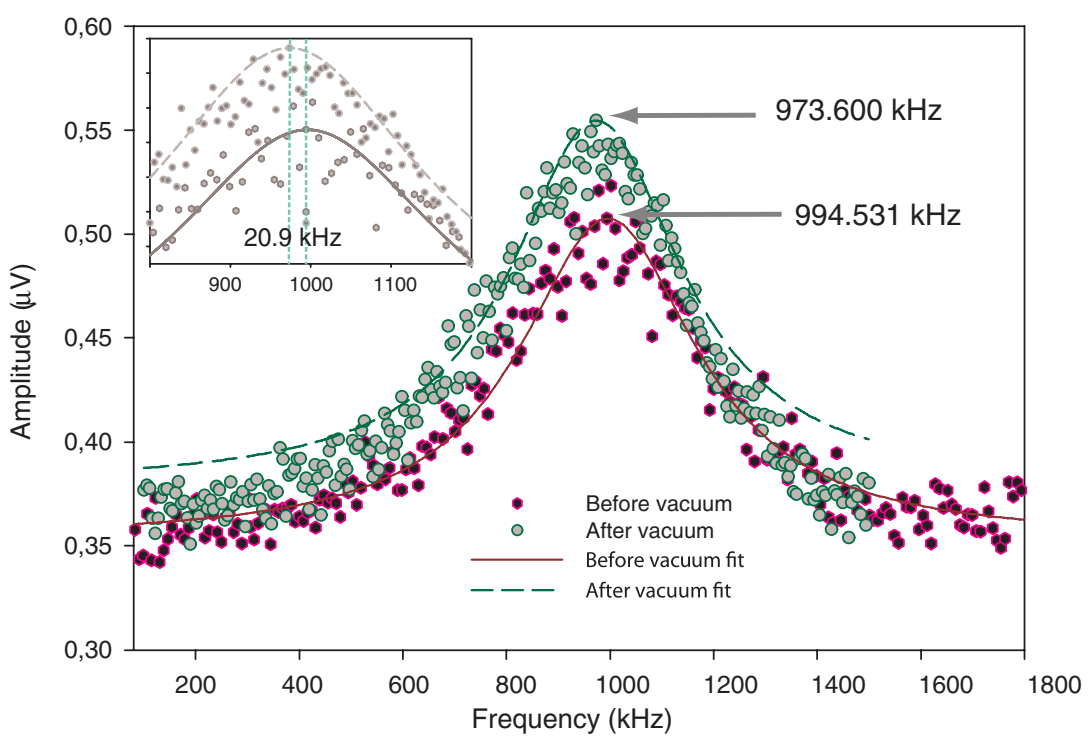

Figure 6. The resonance frequency of a $93 \mathrm{~nm}$ thick cantilever before and after vacuum. The shift in the resonance frequency is $28 \mathrm{kHz}$, which is mainly due to the removal of the water molecules and degassing. The inset shows the zoom-in of the resonance peak.

adsorbed mass and the stiffening effect on the cantilever [7]. Therefore, the true mass of the targeted material or particles cannot be obtained by simple subtraction of the effective mass of contamination. Changes in cantilever stiffness have to be considered as well, and the natural resonance frequency $f_{0}$ in equation (2) can be corrected by means of stiffness measurement, as proposed in [7,31]. The coupling of mass and stiffness can also be observed by comparing the sign changes of $f_{\mathrm{av}}-f_{\mathrm{bv}}$ in table 1 . For thicker cantilevers, the stiffness of the contamination has little effect on the overall stiffness; therefore, the mass effect dominates and the resonance frequency drops in contaminated cantilevers, resulting in positive $f_{\mathrm{av}}-f_{\mathrm{bv}}$. On the other hand, when the cantilever is thin, the effects of the contamination on the stiffness of the cantilever become significant and dominate. Therefore, the resonance frequency increases compared to the clean state, resulting in negative $f_{\mathrm{av}}-f_{\mathrm{bv}}$ for the $93 \mathrm{~nm}$ thick cantilever.

The result from table 1 naturally leads to the question: 'What will happen to the cantilever when it is exposed to the air over the time?' and 'How long does it take for a cantilever resonance frequency to shift from $f_{\mathrm{av}}$ to $f_{\mathrm{bv}}$ ?' We therefore looked at the changes in the resonance frequency over a period of time and present the result in the following.

\subsection{Stability of the resonance frequency in air}

In general, the interactions of the surrounding environment with the cantilever surfaces are time dependent and stabilize after establishment of an equilibrium. In ambient air, the surfaces constantly adsorb and desorb water, hydrogen, hydrocarbons and oxygen. The native oxide, on the other hand, continues to grow until it reaches a few atomic layers $(\sim 2 \mathrm{~nm})$ [32]. Consequently, these effects can generate errors in the output results of the sensors working in the resonance mode, and the long-term behavior of such a sensor becomes an important stability issue. For atmospheric resonator applications, the interactions with the environment result in a time-dependent shift of the resonant properties that is seldom studied. In order to investigate the stability of resonance behavior in air, resonance frequencies of the cantilevers were measured over several hours. Figure 7 shows the results of the measured resonance frequency in air versus time for a $340 \mathrm{~nm}$ thick cantilever. As shown in figure 7, the resonance frequency abruptly drops after venting the vacuum chamber; this is mainly due to the air mass loading effect dissipating energy and slowing the cantilever movements. Afterward, the resonance frequency slowly decreased over $150 \mathrm{~min}$. Between 200 and 800 min the adsorbate molecules locally adsorb at the surface of the cantilever, and then gradually create regions of real layers, which modify the surface stress and consequently the stiffness of the cantilever [33]. The changes in the stiffness of the cantilever will increase the resonance frequency. Finally, the adsorption of the surfaces becomes saturated which results in a constant resonance frequency after approximately 800 min.

\subsection{Laser back-action on cantilever resonance response measurements}

A detection back-action is the disturbances imposed on a device by the detection system during its measurement. In the optical detection system where a laser beam is focused on the tip of the cantilever, heat energy builds up in the device and dissipates slowly, and this can cause changes to mechanical properties of the cantilever and eventually affect the resonance frequency. In this case a mere observation of cantilever resonance becomes a source of error.

The resonance frequency of a device is influenced by the temperature effects, through the temperature dependence of the effective Young's modulus and geometrical dimensions [19, 34, 35]. In particular, silicon cantilever resonators have shown frequency-temperature sensitivity due to the temperature dependence of the effective Young's modulus 


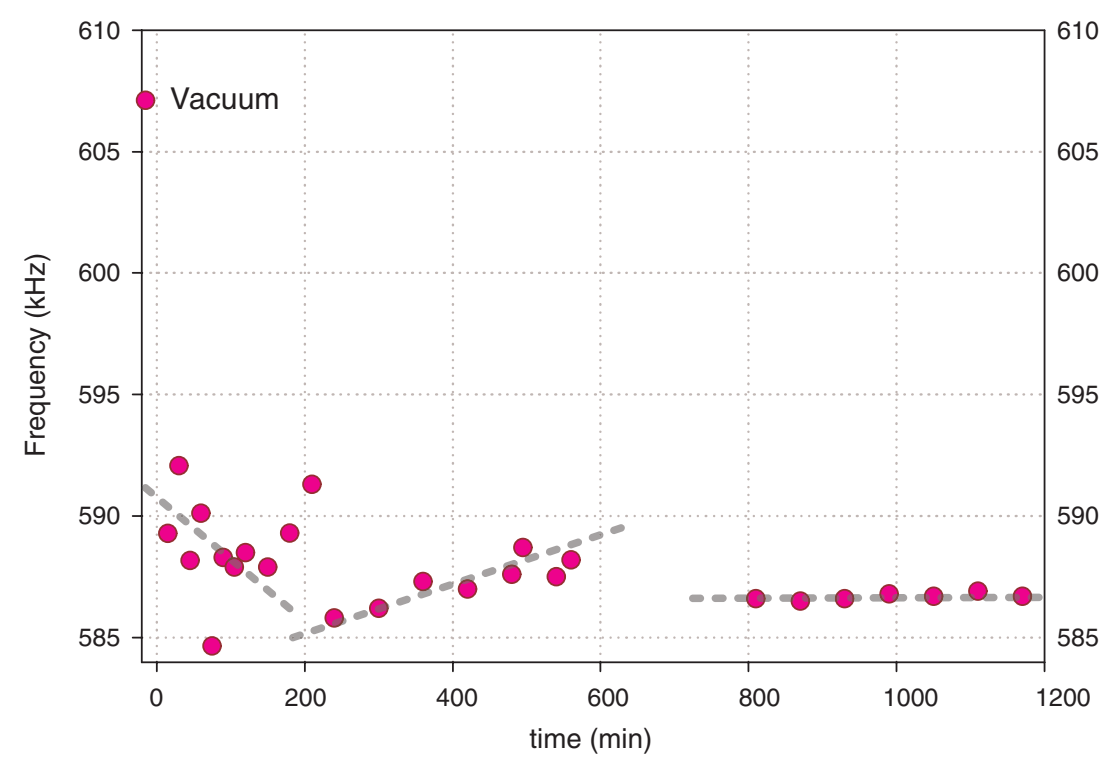

Figure 7. The measured resonance frequency versus time. The measured cantilever is $340 \mathrm{~nm}$ thick, $24 \mu \mathrm{m}$ long and $8 \mu \mathrm{m}$ wide. The dashed lines are visual guides.

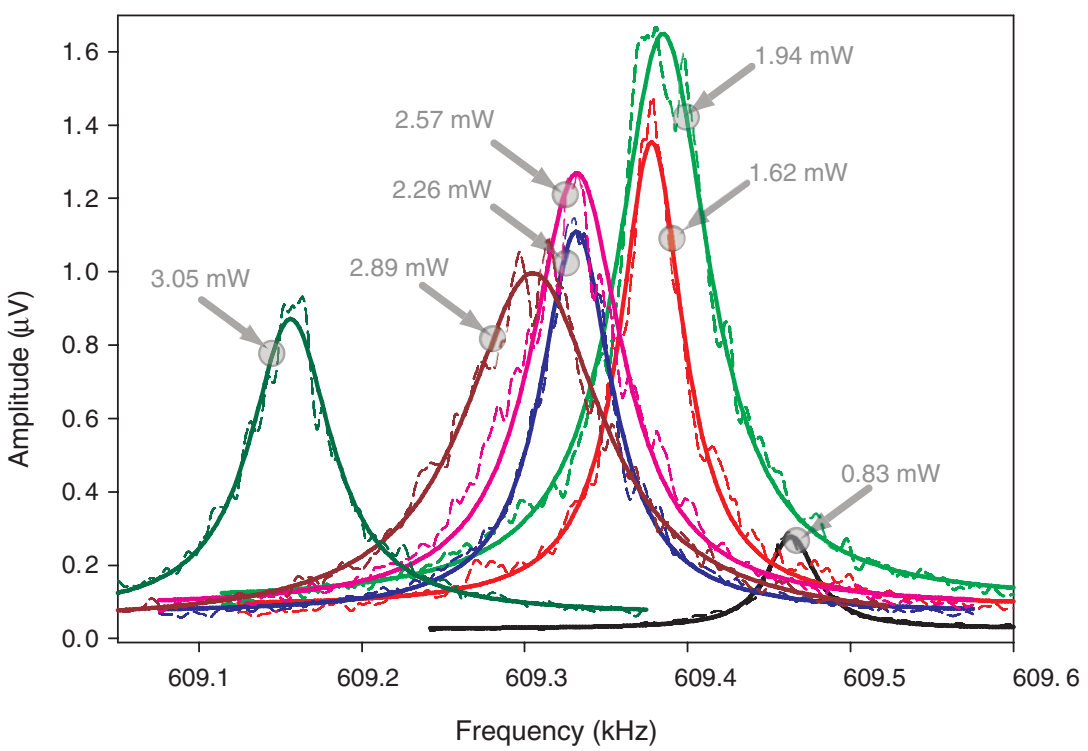

Figure 8. Amplitude versus frequency and the Lorentzian fit for different laser powers. Laser spot at the $24 \mu \mathrm{m}$ long cantilever tip.

[36], which is more than 20 times greater than quartz crystal oscillators [34]. Since the temperature coefficient of the effective Young's modulus of silicon is negative [34, 37], an increase in the temperature results in the material softening and decreases the resonance frequency.

In order to investigate the effect of laser heating, two sets of measurements are carried out: (i) different laser powers are focused on the tips of several cantilevers and the frequency response corresponding to each power for each cantilever is traced. (ii) A fixed laser power is applied to different positions along the length and along the width of the cantilever, and the frequency responses are recorded. All cantilevers are allowed to stabilize for a few minutes prior to each measurement. All measurements are carried out in vacuum to avoid damping, squeeze-film effects, minimizing dissipation of the laser heat and other extrinsic effects in ambient air. The actual laser powers are measured with a power meter under the chamber glass prior to experiments, and the relation between the supplied current (in $\mathrm{mA}$ ) and the power of the laser received by the cantilever is calibrated based on these power meter measurements.

Figure 8 depicts the output voltages, which correspond to vibration amplitudes, versus frequencies and their Lorentzian fits at different laser powers of a $24 \mu \mathrm{m}$ long, $8 \mu \mathrm{m}$ wide and $340 \mathrm{~nm}$ thick cantilever. For thermal noise actuation, $0.83 \mathrm{~mW}$ is the minimum laser power that is needed to cause detectable vibration amplitudes on our set of cantilevers. A sudden amplitude increase is observed in most of the cantilever responses when stepping up the power to $1.62 \mathrm{~mW}$. The different resonance frequencies are clearly visible. The frequency-power measurement is repeated for cantilevers of different lengths with the same width and thickness, and the 


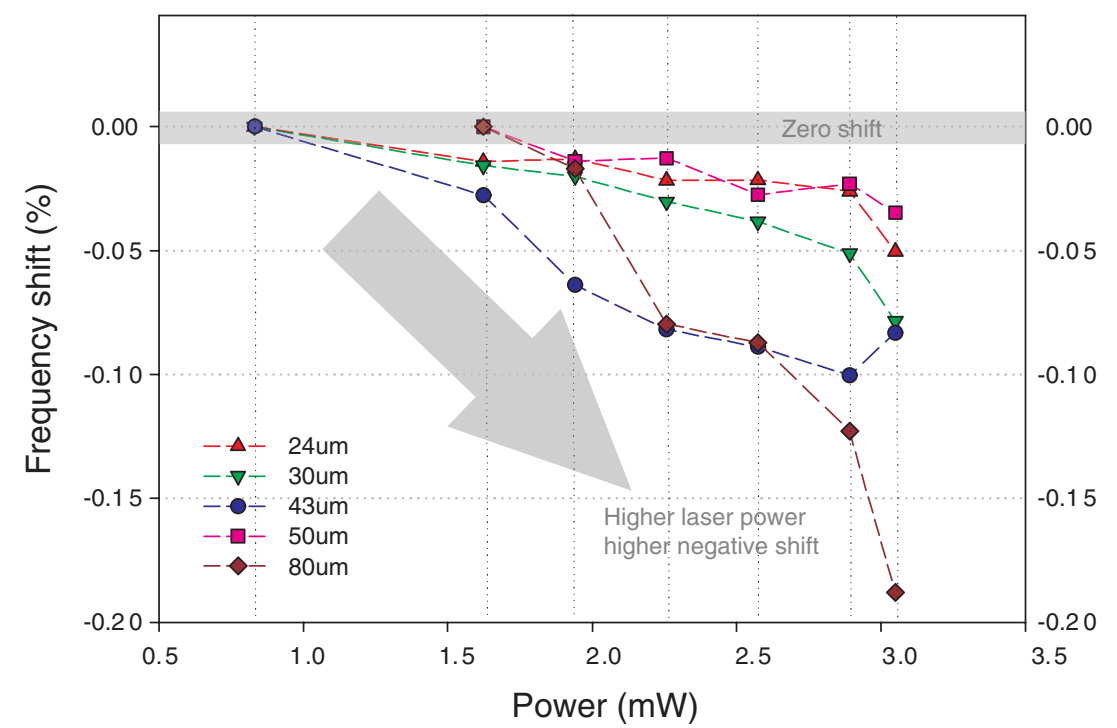

Figure 9. Normalized resonance frequency shift versus laser power. Laser spot at the cantilever tip.

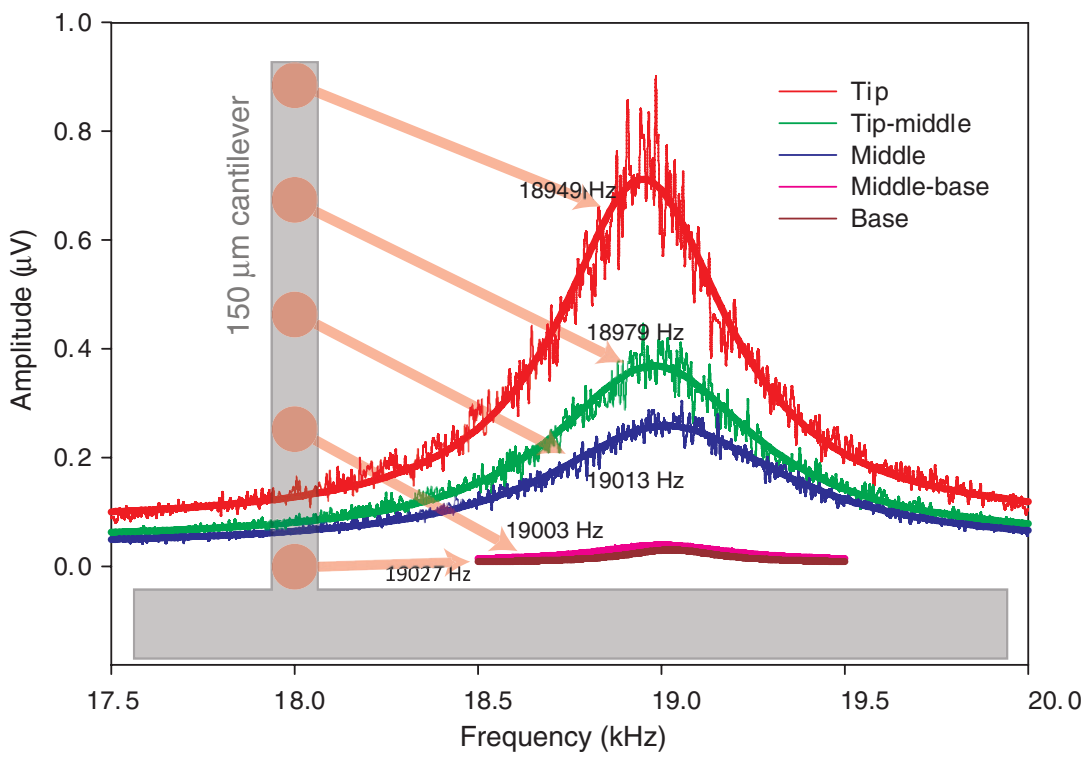

Figure 10. Amplitude versus frequency as a function of different laser spot positions on a $150 \mu \mathrm{m}$ long cantilever.

result is shown in figure 9. In the figure, the frequency measured from the lowest laser power of each cantilever is considered as the base frequency $f_{1}$, and the percentage shift is calculated as $f_{\text {shift }}=\frac{f_{2}-f_{1}}{f_{1}}$. Higher laser powers induce more negative frequency shift and in general, longer cantilevers showed a higher shift at the same power increase compared to the shorter ones. This might indeed indicate that the change in length due to the temperature is important.

In addition to the changes in laser power, the influence of the position of the laser spot was also investigated, namely along the $x$ - and the $y$-axes of the beam. Figure 10 shows the resonance frequency measurement for different laser spot positions of a $150 \mu \mathrm{m}$ cantilever. Figure 11 shows the resonance frequencies from the fitted measurement results versus the spot position on different cantilevers. Zero on the horizontal axis corresponds to the position of the laser spot close to the cantilever base. The frequency shift is calculated by taking the resonance frequency with the spot close to the cantilever base as the base frequency. The frequency shift due to position is a complex coupling of localized joule heating, heat dissipation and clamp softening of the cantilever. When the laser is near the clamping point, heat is dissipated faster through the bulk silicon, but since it is near the base, the localized heating directly on the clamping point is very high. This can induce a larger softening effect, compared to local heating far from clamping point, hence lowering the resonance frequency. On the other hand, when the laser is at the tip end, dissipation is minimum and the whole cantilever is heated up, but it is also further away from the clamping point, resulting in less softening effect. As a result, the shift in the resonance depends on which effect is dominant at a certain position and with a certain power. 


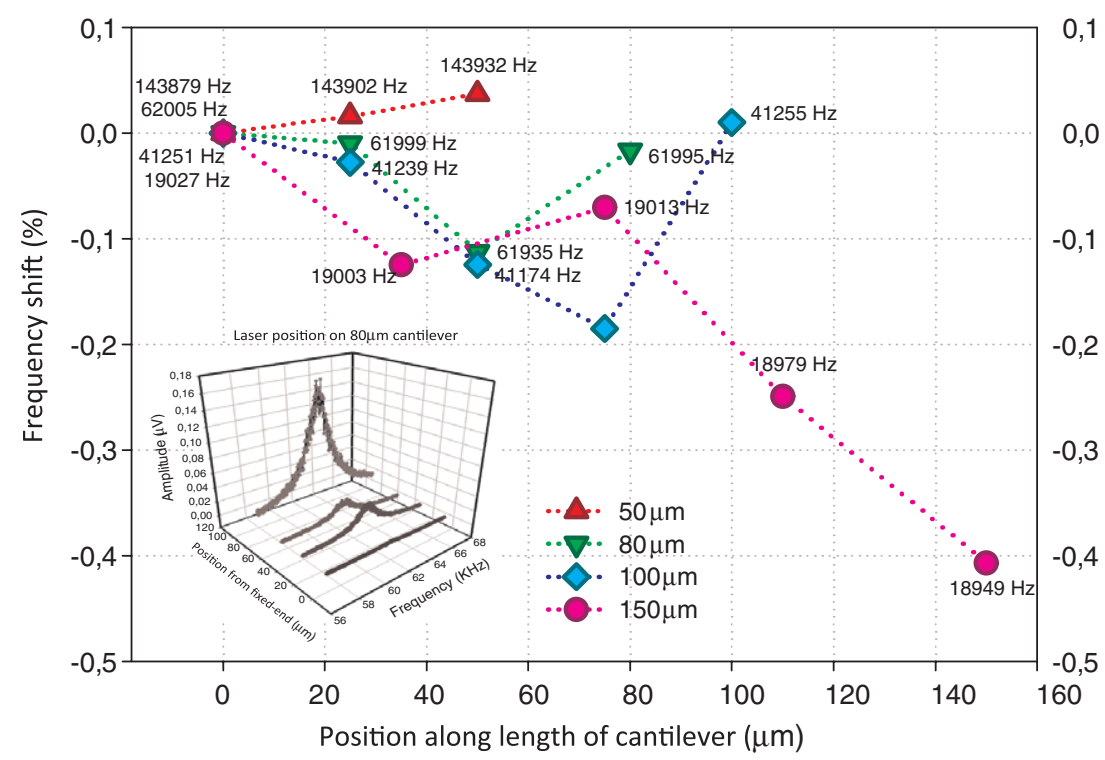

Figure 11. Relative shift in the resonance frequency versus position of the laser spot. The numbers indicate the measured resonance frequency. The inset shows one of the measurements made on the $80 \mu \mathrm{m}$ long cantilever.

Resonance frequencies were also measured along the width ( $y$-axis) of the beam; however, there are no significant changes in the resonance frequency.

Similar to the surface contamination effect, laser measurements can also induce significant resonance frequency shifts, especially changes in the power and the positions along the beam length. Taking the $24 \mu \mathrm{m}$ long cantilever shown in figure 8 as an example, the shift between the $0.83 \mathrm{~mW}$ and $3.05 \mathrm{~mW}$ laser power is $307 \mathrm{~Hz}$. If this cantilever were to be used as a sensitive mass sensor, the laser power effect is enough to induce an effective measurement error of $15.2 \mathrm{pg}$. Although such a large laser power jump is unlikely in a well-controlled setup, for the unprecedented nanocantilever sensing, effects of any small power change still have to be taken into account.

\section{Conclusion}

In conclusion, the resonance behavior of sub-micron silicon cantilevers was characterized in air and vacuum condition. The long-term stability of resonance frequency was studied in air. We showed that although the mass sensitivity of MEMS and NEMS resonant cantilevers is at femtogram level, the specificity is severely degraded by nonspecific adsorption and contamination of the cantilever surface. The equivalent mass of surface contamination was calculated, and was shown to cause significant errors in determining the target mass. On the other hand, the influence of the laser used in an optical detection method, on the resonance characteristics of the cantilevers, was also studied. This included the effect of laser power, and the focused position of the laser spot at the cantilever surface with respect to the clamped point and at the edges. It was shown that the commonly used laser deflection measurements can induce unwanted resonance frequency shifts. Therefore, the choice of using laser deflection for resonance cantilever sensors is not without consequences and the influence of the laser has to be taken into account. Examples in this paper include mass sensing, but it is believed that they can be extended for other sensing applications.

As sensor technology advances, resonant silicon cantilevers will be used more and more often in ultrasensitive measurements. Following this trend, the instability issues caused by the measurement environments and by the detection methods will become an important consideration for the validity of the experiments and the practicality of the device. Future ultra-sensitive measurements should therefore always incorporate discussions on measurements to prevent, or compensate, these resonance instabilities.

\section{Acknowledgments}

This research was supported by the Dutch National Research Program on micro technology, MicroNed (project code: IVC-2), NanoNed (project code TQVB46) and FOM.

\section{References}

[1] Varshney M, Waggoner P S, Tan C P, Aubin K, Montagna R A and Craighead H G 2008 Prion protein detection using nanomechanical resonator arrays and secondary mass labeling Anal. Chem. 80 2141-8

[2] Mamin H J and Rugar D 2001 Sub-attonewton force detection at millikelvin temperatures Appl. Phys. Lett. 79 3358-60

[3] Naik A, Buu O, LaHaye M D, Armour A D, Clerk A A, Blencowe M P and Schwab K C 2006 Cooling a nanomechanical resonator with quantum back-action Nature 443 193-6

[4] Gupta A, Akin D and Bashir R 2004 Single virus particle mass detection using microresonators with nanoscale thickness Appl. Phys. Lett. 84 1976-8

[5] Shen Z, Shih W Y and Shih W-H 2006 Self-exciting, self-sensing $\mathrm{PbZr}_{0.53} \mathrm{Ti}_{0.47} \mathrm{O}_{3} / \mathrm{SiO}_{2}$ piezoelectric microcantilevers with femtogram/hertz sensitivity Appl. Phys. Lett. 89023506 
[6] Yang Y, Callegari C, Feng X, Ekinci K and Roukes M 2006 Zeptogram-scale nanomechanical mass sensing Nano Lett. $6583-6$

[7] Sadeghian H, Goosen H, Bossche A and van Keulen F 2010 Application of electrostatic pull-in instability on sensing adsorbate stiffness in nanomechanical resonators Thin Solid Films 518 5018-21

[8] King W H 1964 Piezoelectric sorption detector Anal. Chem. 36 1735-9

[9] Mecea V M 1994 Loaded vibrating quartz sensors Sensors Actuators A 40 1-27

[10] Kim C-J, Kim J Y and Sridharan B 1998 Comparative evaluation of drying techniques for surface micromachining Sensors Actuators A 64 17-26

[11] Ramos D, Arroyo-HernaÌndez M, Gil-Santos E, Tong H D, Van Rijn C, Calleja M and Tamayo J 2009 Arrays of dual nanomechanical resonators for selective biological detection Anal. Chem. 81 2274-9

[12] Ekinci K 2005 Electromechanical transducers at the nanoscale: actuation and sensing of motion in nanoelectromechanical systems (NEMS) Small 1 786-97

[13] Lavrik N, Sepaniak M J and Datskos P G 2004 Cantilever transducers as a platform for chemical and biological sensors Rev. Sci. Instrum. 75 2229-53

[14] Bell D J, Lu T J, Fleck N A and Spearing S M 2005 MEMS actuators and sensors: observations on their performance and selection for purpose J. Micromech. Microeng. 15 S153

[15] Meyer G and Amer N M 1988 Novel optical approach to atomic force microscopy Appl. Phys. Lett. 53 1045-7

[16] Meyer G and Amer N M 1988 Novel optical approach to atomic force microscopy Appl. Phys. Lett. 53 2400-2 (erratum)

[17] Ilic B, Craighead H G, Krylov S, Senaratne W, Ober C and Neuzil P 2004 Attogram detection using nanoelectromechanical oscillators $J$. Appl. Phys. 95 3694-703

[18] Sone H, Fujinuma Y and Hosaka S 2004 Picogram mass sensor using resonance frequency shift of cantilever Japan. J. Appl. Phys. 43 3648-51

[19] Gysin U, Rast S, Ruff P, Meyer E, Lee D W, Vettiger P and Gerber C 2004 Temperature dependence of the force sensitivity of silicon cantilevers Phys. Rev. B 69045403

[20] Wu G, Ji H, Hansen K, Thundat T, Datar R, Cote R, Hagan M F, Chakraborty A K and Majumdar A 2001 Origin of nanomechanical cantilever motion generated from biomolecular interactions Proc. Natl Acad. Sci. USA 98 1560-4

[21] Braun T, Ghatkesar M K, Backmann N, Grange W, Boulanger P, Letellier L, Lang H-P, Bietsch A, Gerber C and Hegner M 2009 Quantitative time-resolved measurement of membrane protein-ligand interactions using microcantilever array sensors Nat. Nano 4 179-85
[22] Evans D R and Craig V S J 2006 Sensing cantilever beam bending by the optical lever technique and its application to surface stress J. Phys. Chem. B 110 5450-61

[23] Ilic B, Czaplewski D, Zalalutdinov M, Craighead H G, Neuzil P, Campagnolo C and Batt C 2001 Single cell detection with micromechanical oscillators J. Vac. Sci. Technol. B $192825-8$

[24] Duan H, Xue Y and Yi X 2009 Vibration of cantilevers with rough surfaces Acta Mech. Solida Sin. 22 550-4

[25] Weissmüller J and Duan H 2008 Cantilever bending with rough surfaces Phys. Rev. Lett. 101146102

[26] Babaei Gavan K, van der Drift E W J M, Venstra W J, Zuiddam M R and van der Zant H S J 2009 Effect of undercut on the resonant behavior of silicon nitride cantilevers J. Micromech. Microeng. 19 1-8

[27] Sone H, Okano H and Hosaka S 2004 Picogram mass sensor using piezoresistive cantilever for biosensor Japan. J. Appl. Phys. 43 4663-6

[28] Lassagne B, Garcia-Sanchez D, Aguasca A and Bachtold A 2008 Ultrasensitive mass sensing with a nanotube electromechanical resonator Nano Lett. 8 3735-8

[29] Sadeghian H, Yang C K, Babaei Gavan K, Goosen J F L, van der Drift E W J M, van der Zant H S J, French P J, Bossche A and van Keulen F 2009 Effects of surface stress on nanocantilevers $e$-J. Surface Sci. Nanotechnol. 7 161-6

[30] Sone H, Ikeuchi A, Izumi T, Okano H and Hosaka S 2006 Femtogram mass biosensor using self-sensing cantilever for allergy check Japan. J. Appl. Phys. 45 2301-4

[31] Ramos D, Arroyo-HernaÌndez M, Gil-Santos E, Tong H Duy, Van Rijn C, Calleja M and Tamayo J 2009 Arrays of dual nanomechanical resonators for selective biological detection Anal. Chem. 81 2274-9

[32] Gordon M J, Baron T, Dhalluin F, Gentile P and Ferret P 2009 Size effects in mechanical deformation and fracture of cantilevered silicon nanowires Nano Lett. 9 525-9

[33] Kazinczi R 2002 Reliability of micromechanical thin-film resonators $P h D$ Thesis Delft University of Technology

[34] Renata M, Bongsang K, Saurabh A C, Matthew A H, Manu A, Chandra M J and Thomas W K 2007 Temperature-compensated high-stability silicon resonators Appl. Phys. Lett. 90244107

[35] Jianqiang H, Changchun Z, Junhua L and Yongning H 2002 Dependence of the resonance frequency of thermally excited microcantilever resonators on temperature Sensors Actuators A 101 37-41

[36] Sadeghian H, Yang C-K, Goosen H, Bossche A, French P and van Keulen F 2010 Temperature sensitivity of silicon cantilevers' elasticity with the electrostatic pull-in instability Sensors Actuators A $162220-4$

[37] McSkimin H J 1953 Measurement of elastic constants at low temperatures by means of ultrasonic waves-data for silicon and germanium single crystals, and for fused silica J. Appl. Phys. 24 988-97 